\title{
Effect of intraoperative lidocaine on anesthetic consumption, and bowel function, pain intensity, analgesic consumption and hospital stay after breast surgery
}

\author{
Soo Joo Choi, Myung Hee Kim, Hui Yeon Jeong, and Jeong Jin Lee \\ Department of Anesthesiology and Pain Medicine, Samsung Medical Center, Sungkyunkwan University School of Medicine, Seoul, Korea
}

Background: Perioperative lidocaine infusion improves postoperative outcomes, mostly after abdominal and urologic surgeries. Knowledge of the effect of lidocaine on peripheral surgeries is limited. Presently, we investigated whether intraoperative lidocaine infusion reduced anesthetic consumption, duration of ileus, pain intensity, analgesic consumption and hospital stay after breast plastic surgeries.

Methods: Sixty female patients, aged 20-60 years, enrolled in this prospective study were randomly and equally divided to two groups. One group $(\mathrm{n}=30)$ received a $1.5 \mathrm{mg} / \mathrm{kg}$ bolus of lidocaine approximately $30 \mathrm{~min}$ before incision followed by continuous infusion of lidocaine $(1.5 \mathrm{mg} / \mathrm{kg} / \mathrm{h})$ until skin closure (lidocaine group). The other group ( $\mathrm{n}=30)$ was untreated (control group). Balanced inhalation (sevoflurane) anesthesia and multimodal postoperative analgesia were standardized. End tidal sevoflurane concentration during surgery, time to the first flatus and defecation, visual analog pain scale (0-10), analgesic consumption and associated side effects at 24, 48, and 72 $\mathrm{h}$ after surgery, hospital stay, and patient's general satisfaction were assessed.

Results: Compared to the control group, intraoperative lidocaine infusion reduced by $5 \%$ the amount of sevoflurane required at similar bispectral index $(\mathrm{P}=0.014)$. However, there were no significant effects of lidocaine regarding the return of bowel function, postoperative pain intensity, analgesic sparing and side effects at all time points, hospital stay, and level of patient's satisfaction for pain control.

Conclusions: Low dose intraoperative lidocaine infusion offered no beneficial effects on return of bowel function, opioid sparing, pain intensity and hospital stay after various breast plastic surgeries. (Korean J Anesthesiol 2012; 62: 429-434)

Key Words: Analgesic consumption, Anesthetic consumption, Bowel function, Hospital stay, Intravenous lidocaine, Pain score.

Received: August 25, 2011. Revised: September 30, 2011. Accepted: October 11, 2011.

Corresponding author: Myung Hee Kim, M.D., Department of Anesthesiology and Pain Medicine, Samsung Medical Center, Sungkyunkwan University School of Medicine, 50, Ilwon-dong, Gangnam-gu, Seoul 135-710, Korea. Tel: 82-2-3410-2468, Fax: 82-2-3410-0361, E-mail: myungsmc@yahoo.co.kr

(c) This is an open-access article distributed under the terms of the Creative Commons Attribution Non-Commercial License (http:// creativecommons.org/licenses/by-nc/3.0/), which permits unrestricted non-commercial use, distribution, and reproduction in any medium, provided the original work is properly cited. 


\section{Introduction}

Although ileus after surgery often occurs after intraperitoneal surgeries, delayed gastrointestinal motility has been demonstrated after retroperitoneal and peripheral operations [1,2]. Postoperative ileus sometimes can be a potentially extensive and can be of considerable clinical significance, delaying hospital discharge [1,3-5]. A smooth and facilitated postoperative course, including adequate pain control and early oral intake, is often part of contributes to satisfaction in surgical patients. Although the use of opioids remains as the mainstay for postoperative pain control, consumption of opioid-based analgesics can be a potential factor in impairing colonic movement [6]. Thus, adjuvant analgesic therapies have been implemented to reduce the doses of opioids after surgery.

In recent studies, perioperative systemic lidocaine infusion has demonstrated beneficial effects on the early restoration of bowel function, pain control, narcotic sparing and decreased hospital stay, particularly after abdominal and urologic surgeries [1,4,7-9]. Although delayed restoration of bowel activity following surgeries other than the abdominal area has been suggested $[1,2]$, there have been few studies of the perioperative effect of lidocaine on bowel function after peripheral surgery.

As a sodium channel blocker, systemic lidocaine attenuates skin pain in response to mechanical or chemical stimuli through peripheral and central mechanisms in preclinical human volunteer tests [10-12]. However, although the perioperative administration of lidocaine can prevent postoperative pain from various abdominal surgeries, the use of a low dose of lidocaine was not successful on the improvement of postoperative pain control and narcotic reduction in a recent study involving total hip arthroplasty [13].

The positive impact of systemic lidocaine on the postoperative outcomes has been mixed in previous studies. Therefore, we aimed to examine whether intraoperative systemic lidocaine may present beneficial effects on the recovery of bowel function, pain intensity and analgesic consumption in patients undergoing various breast plastic surgeries.

\section{Materials and Methods}

After obtaining our institutional ethics committee approval and written informed consent from each patient, ASA I-II patients $(n=60)$, between the ages of 20 and 60 years, undergoing elective plastic breast surgeries such as aesthetic augmentation mammaplasty, reduction mammaplasty, augmentation mammaplasty with tissue expander removal, breast reconstruction with flap including extended latissimus dorsi (ELD), deep inferior epigastric perforator (DIEP), and transverse rectus abdominis musculocutaneous (TRAM) following immediate or delayed mastectomy or tissue expander insertion following mastectomy with general anesthesia were randomly divided into two groups to receive systemic lidocaine infusion until the end of surgery (lidocaine group, $\mathrm{n}=30$ ) and no infusion (control group, $\mathrm{n}=30$ ) after induction of general anesthesia. For lidocaine group, an intravenous (i.v.) bolus of lidocaine $1.5 \mathrm{mg} / \mathrm{kg}$ was given approximately $30 \mathrm{~min}$ prior to the skin incision, followed by a continuous lidocaine infusion $1.5 \mathrm{mg} / \mathrm{kg} / \mathrm{h}$ throughout surgery until the end of skin closure. Patients enrolled in this study had no severe hepatic, renal, cardiac, respiratory, or endocrine diseases, morbid obesity, or allergies to local anesthetics. The episodes of intraoperative hypotension (mean blood pressure [BP] $<60 \mathrm{mmHg}$ ) or bradycardia (heart rate $[\mathrm{HR}]<40$ beats $/ \mathrm{min}$ ), arrhythmia or urticaria associated with lidocaine infusion were also criteria for exclusion. Surgical variation was limited because two plastic surgeons involved in this study were highly experienced in plastic breast surgeries, and had performed standardized operative techniques according to the surgery types. General anesthesia was induced with midazolam $2 \mathrm{mg}$ and propofol 2 $\mathrm{mg} / \mathrm{kg}$ i.v. under mask ventilation with oxygen and sevoflurane, and rocuronium $0.8 \mathrm{mg} / \mathrm{kg}$ was followed for tracheal intubation. Anesthesia was maintained with sevoflurane with oxygen and medical air $1 \mathrm{~L} / 1 \mathrm{~L}$ during anesthesia. No supplemental opioid was given to patients in either group during the maintenance of anesthesia. Patients were monitored throughout operation with $\mathrm{BP}, \mathrm{HR}$, peripheral oxygen saturation, EKG, core temperature, bispectral index (BIS), end tidal sevoflurane and end tidal $\mathrm{CO}_{2}$. Sevoflurane concentration was adjusted according to the hemodynamic and BIS values. BP and HR were maintained within $20 \%$ of preoperative values and BIS ranged $35-45$ to ensure similar anesthetic depth between the groups. End tidal $\mathrm{CO}_{2}$ was between $35-40 \mathrm{mmHg}$. To maintain core body temperature normothermia, circulating warm water blanket and heated warm circuit were applied. Perioperative fluid was managed with Lactate Ringer's solution at a rate $6-8 \mathrm{ml} / \mathrm{kg}$ / h. At the end of surgery, neuromuscular blockade was reversed with pyridostigmine and glycopyrrolate $(0.3 \mathrm{mg} / \mathrm{kg}$ and 0.015 $\mathrm{mg} / \mathrm{kg}$, respectively). For postoperative pain control, pethidine $0.5 \mathrm{mg} / \mathrm{kg}$ was provided within $30 \mathrm{~min}$ of the end of surgery and supplemented after recovery as needed with further boluses of $0.25 \mathrm{mg} / \mathrm{kg}$ at $20-30 \mathrm{~min}$ intervals. Upon arrival to the post anesthetic care unit (PACU), patients were connected to an intravenous patient controlled analgesic system (IVPCA) with fentanyl 1,500 $\mu \mathrm{g}$ and ketorolac $180 \mathrm{mg}$ in $64 \mathrm{ml}$ of saline (100 $\mathrm{ml}$ of total volume) to deliver a bolus of $1 \mathrm{ml}$ of the above analgesics with a lockout time of $15 \mathrm{~min}$ and a basal rate of 1 $\mathrm{ml} / \mathrm{h}$. During their PACU time, patients were asked to report any side effects of systemic lidocaine such as light-headedness, drowsiness, metal taste, perioral numbness, and visual 
disturbances. After transfer to the ward, all patients received IVPCA, and extra rescue medications such as pethidine or nonsteroid anti-inflammatory drug (NSAID) according to body weight, if required.

Prior to the start of surgery, patients were instructed by a nurse anesthetist how to complete a written self assessment concerning the times to first flatus and first defecation, which indicated restoration of bowel function. The total amount of IVPCA, frequency and total dose of extra analgesics, and opioids associated side effects such as nausea, vomiting, itching, respiratory depression and urinary retention, and pain intensity on a visual analog scale (VAS) ranged $0-10(0=$ no pain, $10=$ most intense pain ever) at 24,48 , and $72 \mathrm{~h}$ after surgery, and the level of satisfaction for pain control at $72 \mathrm{~h}$ after surgery were assessed by a medical investigator. For the safety of patients, anesthesiologists involved in the anesthetic managements were not blinded to the groups. However, they were not involved in further management of postoperative pain control or data collection associated with this study. Participating patients, surgeons, and medical investigators who were involved in the data collection, were all blinded to the patient's group assignment. All patients successfully completed the study. No patient was dropped due to any anesthetic or surgical complication.

The primary end point in the current study was restoration of bowel function after surgery. Power analysis was based on prior data [1], and our own pilot study. Twenty patients per group was necessary to permit a $12 \mathrm{~h}$ difference in the return of bowel function between the two groups, at $\alpha$ level of 0.05 with $80 \%$ power. Consideration to various type of surgeries in the current study, we enrolled patients until 60 patients $(n=30 /$ group $)$ completed the study.

Statistical analysis for comparison between the two groups was conducted using SPSS, version 16 (SPSS Inc, Chicago, IL, USA). Comparison of parametric data and non-parametric data between the two groups was analyzed by using Student's t-test or Mann Whitney rank sum test, depending on distributional properties. Chi-square test or Fisher's exact test was used for the incidence of side effects. Data are expressed as mean \pm standard deviation (SD), median (ranges) or number of patients. $\mathrm{P}<0.05$ was considered statistically significant.

\section{Results}

Vital signs of the patients remained stable at all times during the operation and PACU in either group. No patient in the lidocaine group showed lidocaine associated arrhythmia, severe bradycardia or hypotension during surgery, or delayed recovery from anesthesia. After transfer to the PACU, no patient of the lidocaine group reported subjective symptoms of lidocaine related adverse side effects. Demographic and clinical data, such as surgical types, mean BIS score during surgery, duration of surgery and opioid amount during PACU were comparable between the groups. However, intraoperative anesthetic requirement was significantly less with lidocaine administration. The mean sevoflurane end tidal concentration (from the skin incision to the skin closure) was 5\% lower in the lidocaine group than the control group during the operation (Et sevoflurane, control group $1.95 \pm 0.28 \%$ vs. lidocaine group 1.85 $\pm 0.26 \%, \mathrm{P}=0.014$ ) at similar hemodynamic variables and BIS

Table 1. Demographic Characteristics, Type of Surgery, and Perioperative Data

\begin{tabular}{|c|c|c|c|}
\hline Variables & Control group $(n=30)$ & Lidocaine group $(\mathrm{n}=30)$ & $P$ \\
\hline Age (yr) & $40 \pm 8$ & $41 \pm 10$ & 0.784 \\
\hline Weight (kg) & $55 \pm 7$ & $56 \pm 5$ & 0.599 \\
\hline Height (cm) & $160 \pm 5$ & $160 \pm 1$ & 0.844 \\
\hline \multicolumn{4}{|l|}{ Type of surgery (n) } \\
\hline Augmentation mammaplasty & 5 & 8 & \\
\hline Reduction mammaplasty & 1 & 1 & \\
\hline \multicolumn{4}{|l|}{ TER + Aug.mamaplasty } \\
\hline Single/Both & $5 / 2$ & $3 / 4$ & \\
\hline \multicolumn{4}{|l|}{ Breast reconstruction with flap } \\
\hline ELD/DIEP/TRAM flap & $5 / 5 / 1$ & $6 / 4 / 0$ & \\
\hline Mastectomy with implant & 6 & 4 & \\
\hline Et Sevoflurane (\%) & $1.95 \pm 0.28$ & $1.85 \pm 0.26$ & 0.014 \\
\hline Bispectral index & $39.2 \pm 2.6$ & $38.9 \pm 3.3$ & 0.977 \\
\hline Duration of surgery (min) & $288(210-520)$ & $295(240-500)$ & 0.871 \\
\hline \multicolumn{4}{|l|}{ Opioid requirement during PACU } \\
\hline No of patients & 20 & 17 & \\
\hline Pethidine (mg)/patient & $30.2 \pm 13.3$ & $37.4 \pm 13.1$ & 0.221 \\
\hline
\end{tabular}

Data present mean \pm SD, median (range) or number of patients. TER: tissue expander removal, ELD: extended latissimus dorsi, DIEP: deep inferior epigastric perforator, TRAM: transverse rectus abdominis musculocutaneous, Et: end tidal, PACU: postoperative care unit. 
score (Table 1).

The times to the first flatus and defecation occurred approximately $5 \mathrm{~h}$ and $6 \mathrm{~h}$ faster in the lidocaine group than those of control group, respectively. However, these were not statistically significant differences. Recovery of bowel function after completion of surgery was not benefited by intraoperative small dose lidocaine infusion. When the duration of hospital stay was examined, there was no difference between the two groups (Table 2).

There was no significant difference regarding the effects of lidocaine on pain score, pain rescue analgesic consumption and analgesic associated adverse events at 24,48 and $72 \mathrm{~h}$ after surgery, and level of satisfaction for pain control at $72 \mathrm{~h}$ after surgery, respectively (Table 3 ). Although severe postoperative pain has been noted in a few patients, presently the pain scores

Table 2. Recovery of Bowel Function and Duration of Hospital Stay

\begin{tabular}{lccc}
\hline & $\begin{array}{c}\text { Control group } \\
(\mathrm{n}=30)\end{array}$ & $\begin{array}{c}\text { Lidocaine group } \\
(\mathrm{n}=30)\end{array}$ & $\mathrm{P}$ \\
\hline First flatus (h) & $24.2 \pm 13.7$ & $19.4 \pm 10.4$ & 0.132 \\
First defecation (h) & $69.3 \pm 40.9$ & $63.2 \pm 31.1$ & 0.521 \\
Hospital stay (d) & $11.2 \pm 4.7$ & $11.0 \pm 1.7$ & 0.959 \\
\hline
\end{tabular}

Data are mean \pm SD. were consistent with "mild pain" (average VAS score < 4) for most of the patients in the both groups. Furthermore, a few patients of each group did not require IVPCA for pain control from postoperative day 2. Level of satisfaction for pain control during $72 \mathrm{~h}$ after surgery was presented as more than satisfied in the all patients of the both groups (Table 3 ).

\section{Discussion}

In line with previous studies $[5,8,9]$, the current results demonstrated that intraoperative systemic lidocaine administration of $1.5 \mathrm{mg} / \mathrm{kg} / \mathrm{h}$ until the end of skin closure following a bolus lidocaine of $1.5 \mathrm{mg} / \mathrm{kg}$ as adjunctive anesthetic significantly reduced sevoflurane requirement by $5 \%$ in female patients during a variety of aesthetic and reconstructive breast surgeries at the similar BIS level of control patients. However, we observed no differences between the control group and the lidocaine group subjects regarding postoperative outcomes, such as restoration of bowel function, pain intensity, analgesic consumption, analgesic associated side effects, level of satisfaction for pain control, and the length of hospital stay.

It is important to care for patients without postoperative morbidity to ensure patients satisfaction during the postoperative course. The decreased gastrointestinal motility,

Table 3. Analgesic Use and Side Effects, and VAS Score after Surgery

\begin{tabular}{|c|c|c|c|}
\hline Variables & Control group $(n=30)$ & Lidocaine group $(n=30)$ & $\mathrm{P}$ \\
\hline $24 \mathrm{~h}$ after surgery, $\mathrm{n}$ & 30 & 30 & \\
\hline Total PCA (ml) & $23.8 \pm 16.8$ & $25.5 \pm 14.7$ & 0.861 \\
\hline \multicolumn{4}{|l|}{ Extra pain rescue medication } \\
\hline No. of patients & 1 & 4 & \\
\hline Amount/patient & K30 & $\begin{array}{l}\text { 2: } \mathrm{K} 30+\mathrm{D} 50 \\
\text { 2: D } 50\end{array}$ & \\
\hline VAS score & $3.5 \pm 1.7$ & $3.4 \pm 1.6$ & 0.944 \\
\hline Side effect (N/V/D/I/RD) (n) & $11 / 5 / 3 / 6 / 0$ & $14 / 8 / 1 / 8 / 0$ & NS \\
\hline $48 \mathrm{~h}$ after surgery, $\mathrm{n}$ & 27 & 28 & \\
\hline Total PCA (ml) & $28.8 \pm 15.6$ & $23.8 \pm 16.8$ & 0.683 \\
\hline \multicolumn{4}{|l|}{ Extra pain rescue medication } \\
\hline No of patients & 1 & 1 & \\
\hline Amount/patient & K30 & K30 & \\
\hline VAS score & $3.0 \pm 1.9$ & $2.4 \pm 1.4$ & 0.321 \\
\hline Side effect (N/V/D/I/RD) & $3 / 1 / 2 / 2 / 0$ & $6 / 1 / 1 / 3 / 0$ & NS \\
\hline $72 \mathrm{~h}$ after surgery, $\mathrm{n}$ & 22 & 26 & \\
\hline Total PCA (ml) & $28.5 \pm 13.7$ & $20.9 \pm 17.6$ & 0.221 \\
\hline \multicolumn{4}{|l|}{ Extra pain rescue medication } \\
\hline No of patients & 1 & 1 & \\
\hline Amount/patient & K30 & K30 & \\
\hline VAS score & $2.1 \pm 1.6$ & $1.9 \pm 1.4$ & 0.87 \\
\hline Side effect (N/V/D/I/RD) & $6 / 0 / 1 / 1 / 0$ & $4 / 1 / 1 / 0 / 0$ & NS \\
\hline $\begin{array}{l}\text { Level of satisfaction for pain ce } \\
\text { (excellent/satisfied/poor) }\end{array}$ & $0 / 28 / 0$ & $1 / 29 / 0$ & NS \\
\hline
\end{tabular}

Data are mean \pm SD or number of patients. PCA: patient controlled analgesia. PCA consists of fentanyl 1,500 mcg + ketorolac 180 mg + normal saline $62 \mathrm{ml}$ per $100 \mathrm{ml}$. VAS: visual analog scale. N/V/D/I/RD: nausea/vomiting/itching/respiratory depression.K30: $30 \mathrm{mg}$ of ketorolac as one dose, D50: demerol (pethidine chloride) $50 \mathrm{mg}$. NS: not significant. A few patients of each group did not require IVPCA for pain control from postoperative day 2 . 
associated with nausea, vomiting and delayed oral intake, which may decrease general satisfaction to surgical patients, often occurs after intra-peritoneal surgeries. However, episodes of ileus after extra-peritoneal surgeries were evident $[1,2]$. Although the underlying mechanisms of ileus after surgery were not clarified yet, opioid use [6] for adequate pain control and surge of inflammatory mediators $[4,5]$ in response to surgical stress are thought to be causal factors. Recently, in an effort to minimize postoperative opioids consumption, multimodal coanalgesics have been studied. Perioperative local anesthetic lidocaine infusion as an alternative approach significantly improved outcomes including the spared use of opioids and recovery of bowel function, particularly after abdominal and urologic surgeries [1,4,7-9]. Local anesthetic lidocaine reduces overactive inflammatory responses, which contribute to the development of several bowel diseases. Recently, Herroeder et al. [4] demonstrated that perioperative intravenous lidocaine significantly attenuated plasma levels of pro-inflammatory cytokines, complement and platelet-leukocyte aggregates after colorectal surgery. Furthermore, rapid return of bowel function by perioperative systemic lidocaine infusion was well associated with early hospital discharge after abdominal and urologic surgeries $[1,3,4]$. Interestingly, delay of bowel function following operations other than abdominal area has been described [2]. In that study, a delay of about $16 \mathrm{~h}$ before restoration of bowel activity following peripheral operations has been demonstrated radiologically using radiopaque markers. Although we are unsure as to the effect of perioperative systemic lidocaine infusion on the improvement of bowel function after peripheral surgeries, when considering a delayed gastrointestinal motility after peripheral surgeries, it might be worthwhile to study the effect of systemic lidocaine on the ileus after breast surgeries. Although radiopaque markers were not used, and the reason for the absence of an expected lidocaine effect on the recovery of bowel function was beyond the scope of this study, the time to the first experience of flatus and defecation after surgery were faster average $5 \mathrm{~h}$ and $6 \mathrm{~h}$, respectively, in the lidocaine group subjects compared to control group patients. However, the differences were not statistically significant in this study.

The mechanism of analgesic and antihyperalgesic effect by lidocaine have been explained by suppressing the excessive inputs from peripheral and spinal nerves after trauma in vivo human experiments [10-12]. The effect of systemic lidocaine on the attenuation of pain in response to experimental incision induced pain [11] or intradermal capsacin-induced secondary hyperalgesia $[10,12]$ was demonstrated in the lidocaine treated group of preclinical human volunteer tests. In previous studies with retropubic prostatectomy [1], major abdominal surgery [7], laparoscopic colectomy [8], and laparoscopic cholecystectomy [9], perioperative lidocaine infusion reduced postoperative pain intensity and analgesic consumption. Particularly, the analgesic effect was significant on the third postoperative day after stopping lidocaine infusion in patients undergoing major abdominal surgery, by the preventive property of lidocaine on the induction of central hyperalgesia [7]. In contrast to results reported in abdominal and urologic surgeries with positive analgesic effects, i.v. bolus lidocaine of $1.5 \mathrm{mg} / \mathrm{kg}$ followed by continuous infusion of $3 \mathrm{mg} / \mathrm{kg} / \mathrm{h}$ until the end of surgery failed to demonstrate any benefits for postoperative outcomes in patients after abdominal hysterectomy [14]. The true benefit of systemic lidocaine on the outcomes after abdominal and urologic surgery is uncertain, because systemic lidocaine has been continued over the postoperative periods $1-24 \mathrm{~h}$ after intraoperative infusion with different doses [1,4,7-9]. Reduced postoperative pain and the narcotic sparing effect by the i.v. infusion of lidocaine demonstrated in previous abdominal and urologic studies cannot be extrapolated to the current breast surgeries.

Presently, pain intensity at rest was minor, with a VAS score of approximately 3 , and consumption of PCA was similar between the control group and the lidocaine treated group at 24,48 and $72 \mathrm{~h}$ after surgery. Along with our study, the results of another study [13] demonstrated that low dose perioperative lidocaine (intraoperative lidocaine infusion of $1.5 \mathrm{mg} / \mathrm{kg} / \mathrm{h}$ following a bolus lidocaine of $1.5 \mathrm{mg} / \mathrm{kg}$ over postoperative 1 h) was not effective in controlling either postoperative pain perception or secondary hyperalgesia around surgical wounds after total hip arthroplasty. The authors suggested that the limited postoperative benefit in the hip arthroplasty was likely ascribed to the lower amount of lidocaine and its short duration of use [13]. Indeed, the use of optimal dosage regimens of i.v. lidocaine might have produced different results in this study. When considering the effect of dose and duration of lidocaine infusion $[8,15]$, a possible criticism for the current study with intraoperative low dose of lidocaine may be elicited. However, it should be noted that such a low dose and short duration of lidocaine infusion with a $1.5 \mathrm{mg} / \mathrm{kg}$ of lidocaine bolus $30 \mathrm{~min}$ before incision followed by $1.5 \mathrm{mg} / \mathrm{kg} / \mathrm{h}$ lidocaine infusion until $1 \mathrm{~h}$ after skin closure previously demonstrated a significant and prolonged improvement in postoperative pain intensity and analgesic consumption in a study with major abdominal surgery [7].

Nevertheless, the analgesic property of lidocaine seems to depend on the dose infused. Tremont-Lukats et al. [15] reported that the higher dose of lidocaine, $5 \mathrm{mg} / \mathrm{kg}$, was more effective in alleviating neuropathic pain compared to lower infusion, which was not different from placebo for relief of pain.

Although systemic lidocaine administration was well tolerated without significant complications in previous studies $[1,4,7-9]$, a larger dose of lidocaine may induce systemic toxicity. 
There is a scarcity of studies that systemically assessed the safety of i.v. lidocaine. Higher incidence of side effects such as perioral numbness and tinnitus was observed in lidocaine treatment alone [16]. Close medical observation, which is labor intensive and costly, may be required as long as i.v. lidocaine infusion is being continued. In addition, as with the current study, by stopping lidocaine infusion at the end of surgery, it is possible to obtain a lidocaine induced benefit when patients are not receiving drug. Further research to determine optimal dose and duration of systemic lidocaine infusion providing postoperative benefits is necessary in patients undergoing breast surgeries.

In conclusion, the present study did not show any benefit of the intraoperative low dose lidocaine in terms of functional recovery of bowel movement, pain intensity, analgesic consumption and hospital stay after aesthetic and reconstructive breast surgeries.

\section{References}

1. Groudine SB, Fisher HA, Kaufman RP Jr, Patel MK, Wilkins LJ, Mehta SA, et al. Intravenous lidocaine speeds the return of bowel function, decreases postoperative pain, and shortens hospital stay in patients undergoing radical retropubic prostatectomy. Anesth Analg 1998; 86: 235-9.

2. Wilson JP. Postoperative motility of the large intestine in man. Gut 1975; 16: 689-92.

3. Harvey KP, Adair JD, Isho M, Robinson R. Can intravenous lidocaine decrease postsurgical ileus and shorten hospital stay in elective bowel surgery? A pilot study and literature review. Am J Surg 2009; 198: 231-6.

4. Herroeder S, Pecher S, Schonherr ME, Kaulitz G, Hahnenkamp K, Friess $\mathrm{H}$, et al. Systemic lidocaine shortens length of hospital stay after colorectal surgery: a double-blinded, randomized, placebocontrolled trial. Ann Surg 2007; 246: 192-200.

5. Kuo CP, Jao SW, Chen KM, Wong CS, Yeh CC, Sheen MJ, et al. Comparison of the effects of thoracic epidural analgesia and i.v. infusion with lidocaine on cytokine response, postoperative pain and bowel function in patients undergoing colonic surgery. Br J Anaesth 2006;
97: 640-6.

6. White PF. The role of non-opioid analgesic techniques in the management of pain after ambulatory surgery. Anesth Analg 2002; 94: 577-85.

7. Koppert W, Weigand M, Neumann F, Sittl R, Schuettler J, Schmelz $\mathrm{M}$, et al. Perioperative intravenous lidocaine has preventive effects on postoperative pain and morphine consumption after major abdominal surgery. Anesth Analg 2004; 98: 1050-5.

8. Kaba A, Laurent SR, Detroz BJ, Sessler DI, Durieux ME, Lamy ML, et al. Intravenous lidocaine infusion facilitates acute rehabilitation after laparoscopic colectomy. Anesthesiology 2007; 106: 11-8.

9. Lauwick S, Kim do J, Michelagnoli G, Mistraletti G, Feldman L, Fried G, et al. Intraoperative infusion of lidocaine reduces postoperative fentanyl requirements in patients undergoing laparoscopic cholecystectomy. Can J Anaesth 2008; 55: 754-60.

10. Dirks J, Fabricius P, Petersen KL, Rowbotham MC, Dahl JB. The effect of systemic lidocaine on pain and secondary hyperalgesia associated with the heat/capsaicin sensitization model in healthy volunteers. Anesth Analg 2000; 91: 967-72.

11. Kawamata M, Takahashi T, Kozuka Y, Nawa Y, Nishikawa K, Narimatsu E, et al. Experimental incision-induced pain in human skin: effects of systemic lidocaine on flare formation and hyperalgesia. Pain 2002; 100: 77-89.

12. Koppert W, Ostermeier N, Sittl R, Weidner C, Schmelz M. Low-dose lidocaine reduces secondary hyperalgesia by a central mode of action. Pain 2000; 85: 217-24.

13. Martin F, Cherif K, Gentili ME, Enel D, Abe E, Alvarez JC, et al. Lack of impact of intravenous lidocaine on analgesia, functional recovery, and nociceptive pain threshold after total hip arthroplasty. Anesthesiology 2008; 109: 118-23.

14. Bryson GL, Charapov I, Krolczyk G, Taljaard M, Reid D. Intravenous lidocaine does not reduce length of hospital stay following abdominal hysterectomy. Can J Anaesth 2010; 57: 759-66.

15. Tremont-Lukats IW, Hutson PR, Backonja MM. A randomized, double-masked, placebo-controlled pilot trial of extended IV lidocaine infusion for relief of ongoing neuropathic pain. Clin J Pain 2006; $22:$ 266-71.

16. Sharma S, Rajagopal MR, Palat G, Singh C, Haji AG, Jain D. A phase II pilot study to evaluate use of intravenous lidocaine for opioidrefractory pain in cancer patients. J Pain Symptom Manage 2009; 37: 85-93. 